\title{
THE FIBRE OF THE DEGREE 3 MAP, ANICK SPACES AND THE DOUBLE SUSPENSION
}

\author{
STEVEN AMELOTTE
}

\begin{abstract}
Let $S^{2 n+1}\{p\}$ denote the homotopy fibre of the degree $p$ self map of $S^{2 n+1}$. For primes $p \geq 5$, work of Selick shows that $S^{2 n+1}\{p\}$ admits a nontrivial loop space decomposition if and only if $n=1$ or $p$. Indecomposability in all but these dimensions was obtained by showing that a nontrivial decomposition of $\Omega S^{2 n+1}\{p\}$ implies the existence of a $p$-primary Kervaire invariant one element of order $p$ in $\pi_{2 n(p-1)-2}^{S}$. We prove the converse of this last implication and observe that the homotopy decomposition problem for $\Omega S^{2 n+1}\{p\}$ is equivalent to the strong $p$-primary Kervaire invariant problem for all odd primes. For $p=3$, we use the 3-primary Kervaire invariant element $\theta_{3}$ to give a new decomposition of $\Omega S^{55}\{3\}$ analogous to Selick's decomposition of $\Omega S^{2 p+1}\{p\}$ and as an application prove two new cases of a long-standing conjecture stating that the fibre of the double suspension $S^{2 n-1} \longrightarrow \Omega^{2} S^{2 n+1}$ is homotopy equivalent to the double loop space of Anick's space.
\end{abstract}

\section{INTRODUCTION}

Localize all spaces and maps at an odd prime $p$. Let $S^{2 n+1}\{p\}$ denote the homotopy fibre of the degree $p$ map on $S^{2 n+1}$ and let $W_{n}$ denote the homotopy fibre of the double suspension $E^{2}: S^{2 n-1} \longrightarrow \Omega^{2} S^{2 n+1}$. In [20] and [21], Selick showed that there is a homotopy decomposition

$$
\Omega^{2} S^{2 p+1}\{p\} \simeq \Omega^{2} S^{3}\langle 3\rangle \times W_{p},
$$

where $S^{3}\langle 3\rangle$ is the 3 -connected cover of $S^{3}$, and obtained as an immediate corollary that $p$ annihilates all $p$-torsion in $\pi_{*}\left(S^{3}\right)$. This exponent result is generalized by the exponent theorem of Cohen, Moore and Neisendorfer [6, 7, 16], who used different loop space decompositions to construct a map $\varphi: \Omega^{2} S^{2 n+1} \longrightarrow S^{2 n-1}$ with the property that the composite

$$
\Omega^{2} S^{2 n+1} \stackrel{\varphi}{\longrightarrow} S^{2 n-1} \stackrel{E^{2}}{\longrightarrow} \Omega^{2} S^{2 n+1}
$$

is homotopic to the $p^{\text {th }}$ power map on $\Omega^{2} S^{2 n+1}$ and proved by induction on $n$ that $p^{n}$ annihilates the $p$-torsion in $\pi_{*}\left(S^{2 n+1}\right)$. By a result of Gray [10], if $p$ is an odd prime, then $\pi_{*}\left(S^{2 n+1}\right)$ contains infinitely many elements of order $p^{n}$, so this is the best possible odd primary homotopy exponent for spheres. The work of Cohen, Moore and Neisendorfer suggested that there should exist a space $T^{2 n+1}(p)$ fitting in a fibration sequence

$$
\Omega^{2} S^{2 n+1} \stackrel{\varphi}{\longrightarrow} S^{2 n-1} \longrightarrow T^{2 n+1}(p) \longrightarrow \Omega S^{2 n+1}
$$

in which their map $\varphi$ occurs as the connecting map. The existence of such a fibration was first proved by Anick for $p \geq 5$ in [2]. A much simpler construction, valid for all odd primes, was later

2010 Mathematics Subject Classification. 55P35, 55P10.

Key words and phrases. loop space decomposition, double suspension, Anick space, Kervaire invariant. 
given by Gray and Theriault in [15], in which they also show that Anick's space $T^{2 n+1}(p)$ has the structure of an $H$-space and that all maps in the fibration above can be chosen to be $H$-maps.

A well-known conjecture in unstable homotopy theory states that the fibre $W_{n}$ of the double suspension $E^{2}: S^{2 n-1} \longrightarrow \Omega^{2} S^{2 n+1}$ is a double loop space. Anick's space represents a potential candidate for a double classifying space of $W_{n}$, and one of Cohen, Moore and Neisendorfer's remaining open conjectures in [8] states that there should be a $p$-local homotopy equivalence $W_{n} \simeq \Omega^{2} T^{2 n p+1}(p)$. A stronger form of the conjecture (see e.g. [3], [12, [26]) states that

$$
B W_{n} \simeq \Omega T^{2 n p+1}(p)
$$

where $B W_{n}$ is the classifying space of $W_{n}$ first constructed by Gray [11. Such equivalences have only been shown to exist for $n=1$ and $n=p$. In the former case, both $B W_{1}$ and $\Omega T^{2 p+1}(p)$ are known to be homotopy equivalent to $\Omega^{2} S^{3}\langle 3\rangle$. Using Anick's fibration, Selick showed in 23 that $T^{2 p+1}(p) \simeq \Omega S^{3}\langle 3\rangle$ and that the decomposition (11) can be delooped to a homotopy equivalence

$$
\Omega S^{2 p+1}\{p\} \simeq \Omega S^{3}\langle 3\rangle \times B W_{p} .
$$

The $n=p$ case was proved in the strong form $B W_{p} \simeq \Omega T^{2 p^{2}+1}(p)$ by Theriault [26] using the above decomposition in an essential way. Under these identifications, he further showed that $\Omega S^{2 p+1}\{p\}$ and $T^{2 p+1}(p) \times \Omega T^{2 p^{2}+1}(p)$ are equivalent as $H$-spaces.

For primes $p \geq 5$, similar decompositions of $\Omega S^{2 n+1}\{p\}$ are not possible if $n \neq 1$ or $p$. This result was obtained in [22] by first showing that for $n>1$ the existence of a certain spherical homology class imposed by a nontrivial homotopy decomposition of $\Omega S^{2 n+1}\{p\}$ implies the existence of an element of $p$-primary Kervaire invariant one in $\pi_{2 n(p-1)-2}^{S}$, and then appealing to Ravenel's [18] result on the nonexistence of such elements when $p \geq 5$ and $n \neq p$. For $p=3$, the question of whether $\Omega S^{2 n+1}\{3\}$ admits a nontrivial decomposition for $n=3^{j}$ with $j>1$ was left open. In this short note, we prove that the strong odd primary Kervaire invariant problem is in fact equivalent to the problem of decomposing the loop space $\Omega S^{2 n+1}\{p\}$. When $p=3$, this equivalence can be used to import results from stable homotopy theory to obtain new results concerning the unstable homotopy type of $\Omega S^{2 n+1}\{3\}$ as well as some cases of the conjecture that $W_{n}$ is a double loop space.

Theorem 1.1. Let $p$ be an odd prime. Then the following are equivalent:

(a) There exists a p-primary Kervaire invariant one element $\theta_{j} \in \pi_{2 p^{j}(p-1)-2}^{S}$ of order $p$;

(b) There is a homotopy decomposition of $H$-spaces

$$
\Omega S^{2 p^{j}+1}\{p\} \simeq T^{2 p^{j}+1}(p) \times \Omega T^{2 p^{j+1}+1}(p) .
$$

Furthermore, if the above conditions hold, then there are homotopy equivalences of $H$-spaces

$$
B W_{p^{j-1}} \simeq \Omega T^{2 p^{j}+1}(p) \text { and } B W_{p^{j}} \simeq \Omega T^{2 p^{j+1}+1}(p) .
$$

From this point of view, Selick's decomposition of $\Omega S^{2 p+1}\{p\}$ and the previously known equivalences $B W_{1} \simeq \Omega T^{2 p+1}(p)$ and $B W_{p} \simeq \Omega T^{2 p^{2}+1}(p)$ correspond to the existence (at all odd primes) of the Kervaire invariant class $\theta_{1}=\beta_{1} \in \pi_{2 p^{2}-2 p-2}^{S}$ given by the first element of the periodic beta family in the stable homotopy groups of spheres. By Ravenel's negative solution to the Kervaire 
invariant problem for primes $p \geq 5$, Theorem 1.1 has new content only at the prime $p=3$. For example, in addition to the 3-primary Kervaire invariant element $\theta_{1} \in \pi_{10}^{S}$ for $p=3$ and $j=1$ corresponding to the decomposition of $\Omega S^{7}\{3\}$, it is known that there exists a 3-primary Kervaire invariant element $\theta_{3} \in \pi_{106}^{S}$ which we use to obtain the following decomposition of $\Omega S^{55}\{3\}$ and prove the $n=p^{2}$ and $n=p^{3}$ cases of the $B W_{n} \simeq \Omega T^{2 n p+1}(p)$ conjecture at $p=3$.

Corollary 1.2. There are 3 -local homotopy equivalences of $H$-spaces

(a) $\Omega S^{55}\{3\} \simeq T^{55}(3) \times \Omega T^{163}(3)$

(b) $B W_{9} \simeq \Omega T^{55}(3)$

(c) $B W_{27} \simeq \Omega T^{163}(3)$.

Remark 1.3. The equivalence of conditions (a) and (b) in Theorem 1.1 does not hold for $p=2$. In 4, Campbell, Cohen, Peterson and Selick showed that for $n>1$ a nontrivial decomposition of the fibre $\Omega^{2} S^{2 n+1}\{2\}$ of the squaring map implies the existence of an element $\theta \in \pi_{2 n-2}^{S}$ of Kervaire invariant one such that $\theta \eta$ is divisible by 2 . Since such elements are well known to exist only for $n=2,4$ or 8 , these are the only dimensions for which $\Omega^{2} S^{2 n+1}\{2\}$ can decompose nontrivially. Explicit decompositions of $\Omega^{2} S^{5}\{2\}, \Omega^{2} S^{9}\{2\}$ and $\Omega^{3} S^{17}\{2\}$ corresponding to the first three 2primary Kervaire invariant classes $\theta_{1}=\eta^{2}, \theta_{2}=\nu^{2}$ and $\theta_{3}=\sigma^{2}$ are given in [5], [9] and [1].

A further consequence of Theorem 1.1 unique to the $p=3$ case concerns the associativity of Anick spaces. Unlike when $p \geq 5$, in which case $T^{2 n+1}(p)$ is a homotopy commutative and homotopy associative $H$-space for all $n \geq 1$, counterexamples to the homotopy associativity of $T^{2 n+1}(3)$ have been observed in [13] and [24]. In particular, in [13, it was shown that if $T^{2 n+1}(3)$ is homotopy associative, then $n=3^{j}$ for some $j \geq 0$. The proof given there also shows that a homotopy associative $H$-space structure on $T^{2 n+1}(3)$ implies the existence of a three-cell complex

$$
S^{2 n+1} \cup_{3} e^{2 n+2} \cup e^{6 n+1}
$$

with nontrivial mod 3 Steenrod operation $\mathcal{P}^{n}$, which in turn implies (by Spanier-Whitehead duality and the Liulevicius-Shimada-Yamanoshita factorization of $\mathcal{P}^{p^{j}}$ by secondary cohomology operations) the existence of an element of strong Kervaire invariant one. Using Theorem [1.1, we observe that the converse is also true to obtain the following.

Theorem 1.4. Let $n>1$. Then the mod 3 Anick space $T^{2 n+1}(3)$ is homotopy associative if and only if there exists a 3-primary Kervaire invariant one element of order 3 in $\pi_{4 n-2}^{S}$.

\section{The Proof of Theorem 1.1}

The bulk of the proof of Theorem 1.1 will consist of a slight generalization of the argument given in [26. Theorem 1.2], which we briefly describe below. As in [26, the following extension lemma, originally proved in $[3$ for $p \geq 5$ and later extended to include the $p=3$ case in [15, will be crucial. We write $P^{n}\left(p^{r}\right)$ for the mod $p^{r}$ Moore space $S^{n-1} \cup_{p^{r}} e^{n}$ and for a space $X$ define homotopy groups with $\mathbb{Z} / p^{r} \mathbb{Z}$ coefficients by $\pi_{n}\left(X ; \mathbb{Z} / p^{r} \mathbb{Z}\right)=\left[P^{n}\left(p^{r}\right), X\right]$. 
Lemma 2.1. Let $p$ be an odd prime. Let $X$ be an $H$-space such that $p^{k} \cdot \pi_{2 n p^{k}-1}\left(X ; \mathbb{Z} / p^{k+1} \mathbb{Z}\right)=0$ for $k \geq 1$. Then any map $P^{2 n}(p) \rightarrow X$ extends to a map $T^{2 n+1}(p) \rightarrow X$.

In [15], Anick's space is constructed as the homotopy fibre in a secondary EHP fibration

$$
T^{2 n+1}(p) \stackrel{E}{\longrightarrow} \Omega S^{2 n+1}\{p\} \stackrel{H}{\longrightarrow} B W_{n}
$$

where $E$ is an $H$-map which induces in $\bmod p$ homology the inclusion of

$$
H_{*}\left(T^{2 n+1}(p)\right) \cong \Lambda\left(a_{2 n-1}\right) \otimes \mathbb{Z} / p \mathbb{Z}\left[c_{2 n}\right]
$$

into

$$
H_{*}\left(\Omega S^{2 n+1}\{p\}\right) \cong\left(\bigotimes_{i=0}^{\infty} \Lambda\left(a_{2 n p^{i}-1}\right)\right) \otimes\left(\bigotimes_{i=1}^{\infty} \mathbb{Z} / p \mathbb{Z}\left[b_{2 n p^{i}-2}\right]\right) \otimes \mathbb{Z} / p \mathbb{Z}\left[c_{2 n}\right],
$$

and $H$ induces the projection onto

$$
H_{*}\left(B W_{n}\right) \cong\left(\bigotimes_{i=1}^{\infty} \Lambda\left(a_{2 n p^{i}-1}\right)\right) \otimes\left(\bigotimes_{i=1}^{\infty} \mathbb{Z} / p \mathbb{Z}\left[b_{2 n p^{i}-2}\right]\right) .
$$

When $n=p$, it follows from Selick's decomposition of $\Omega S^{2 p+1}\{p\}$ that $H$ admits a right homotopy inverse $s: B W_{p} \rightarrow \Omega S^{2 p+1}\{p\}$ splitting the homotopy fibration (2) in this case. Restricting to the bottom cell of $B W_{p}$, Theriault [26] extended the composite

$$
S^{2 p^{2}-2} \longleftrightarrow B W_{p} \stackrel{s}{\longrightarrow} \Omega S^{2 p+1}\{p\}
$$

to a map $P^{2 p^{2}-1}(p) \rightarrow \Omega S^{2 p+1}\{p\}$ and then applied Lemma 2.1 to the adjoint map $P^{2 p^{2}}(p) \rightarrow$ $S^{2 p+1}\{p\}$ to obtain an extension $T^{2 p^{2}+1}(p) \rightarrow S^{2 p+1}\{p\}$. Finally, looping this last map, he showed that the composite

$$
\Omega T^{2 p^{2}+1}(p) \longrightarrow \Omega S^{2 p+1}\{p\} \stackrel{H}{\longrightarrow} B W_{p}
$$

is a homotopy equivalence, thus proving the $n=p$ case of the conjecture that $B W_{n} \simeq \Omega T^{2 n p+1}(p)$.

In our case, we will use Lemma 2.1 to first construct a right homotopy inverse of $H: \Omega S^{2 n+1}\{p\} \rightarrow$ $B W_{n}$ in dimensions $n=p^{j}$ for which there exists an element $\theta_{j} \in \pi_{2 p^{j}(p-1)-2}^{S}$ of strong Kervaire invariant one and then follow the same strategy as above to obtain both a homotopy decomposition of $\Omega S^{2 p^{j}+1}\{p\}$ and a homotopy equivalence $B W_{p^{j}} \simeq \Omega T^{2 p^{j+1}+1}(p)$. These equivalences can then be used to compare the loops on (2) with the $n=p^{j-1}$ case of a homotopy fibration

$$
B W_{n} \longrightarrow \Omega^{2} S^{2 n p+1}\{p\} \longrightarrow W_{n p}
$$

to further obtain a homotopy equivalence of fibres $B W_{p^{j-1}} \simeq \Omega T^{2 p^{j}+1}(p)$.

Proof of Theorem 1.1. We first show that condition (b) implies condition (a). Given any homotopy equivalence

$$
\psi: T^{2 p^{j}+1}(p) \times \Omega T^{2 p^{j+1}+1}(p) \stackrel{\sim}{\longrightarrow} \Omega S^{2 p^{j}+1}\{p\},
$$

set $n=p^{j}$ and let $f$ denote the composite

$$
f: S^{2 n p-2} \longleftrightarrow \Omega T^{2 n p+1}(p) \stackrel{i_{2}}{\longrightarrow} T^{2 n+1}(p) \times \Omega T^{2 n p+1}(p) \stackrel{\psi}{\longrightarrow} \Omega S^{2 n+1}\{p\}
$$


where the first map is the inclusion of the bottom cell of $\Omega T^{2 n p+1}(p)$ and the second map $i_{2}$ is the inclusion of the second factor. Then

$$
f_{*}(\iota)=b_{2 n p-2} \in H_{2 n p-2}\left(\Omega S^{2 n+1}\{p\}\right)
$$

where $\iota$ is the generator of $H_{2 n p-2}\left(S^{2 n p-2}\right)$. Since the homology class $b_{2 n p-2}$ is spherical if and only if there exists a stable map $g: P^{2 n(p-1)-1}(p) \rightarrow S^{0}$ such that the Steenrod operation $\mathcal{P}^{n}$ acts nontrivially on $H^{*}\left(C_{g}\right)$ by [22], it follows that $\pi_{2 n(p-1)-2}^{S}$ contains an element of $p$-primary Kervaire invariant one and order $p$.

Conversely, suppose there exists a $p$-primary Kervaire invariant one element $\theta_{j} \in \pi_{2 p^{j}(p-1)-2}^{S}$ of order $p$. Then by [22], the homology class $b_{2 p^{j+1}-2} \in H_{2 p^{j+1}-2}\left(\Omega S^{2 p^{j}+1}\{p\}\right)$ is spherical, so there exists a map $f: S^{2 p^{j+1}-2} \rightarrow \Omega S^{2 p^{j}+1}\{p\}$ with Hurewicz image $b_{2 p^{j+1}-2}$. Now following the proof of [26, Theorem 1.2], since $\Omega S^{2 p^{j}+1}\{p\}$ has $H$-space exponent $p$, it follows that $f$ has order $p$ and hence extends to a map

$$
e: P^{2 p^{j+1}-1}(p) \longrightarrow \Omega S^{2 p^{j}+1}\{p\} .
$$

Let $\hat{e}: P^{2 p^{j+1}}(p) \rightarrow S^{2 p^{j}+1}\{p\}$ denote the adjoint of $e$. Again, because $\Omega S^{2 p^{j}+1}\{p\}$ has $H$-space exponent $p$, we have that

$$
p \cdot \pi_{*}\left(S^{2 p^{j}+1}\{p\} ; \mathbb{Z} / p^{k} \mathbb{Z}\right)=0
$$

for all $k \geq 1$, and since $S^{2 p^{j}+1}\{p\}$ is an $H$-space [17, the map $\hat{e}$ satisfies the hypotheses of Lemma 2.1 and therefore admits an extension

$$
s: T^{2 p^{j+1}+1}(p) \longrightarrow S^{2 p^{j}+1}\{p\} .
$$

Note that this factorization of $\hat{e}$ through $s$ implies that the adjoint map $e$ factors through $\Omega s$, so we have a commutative diagram

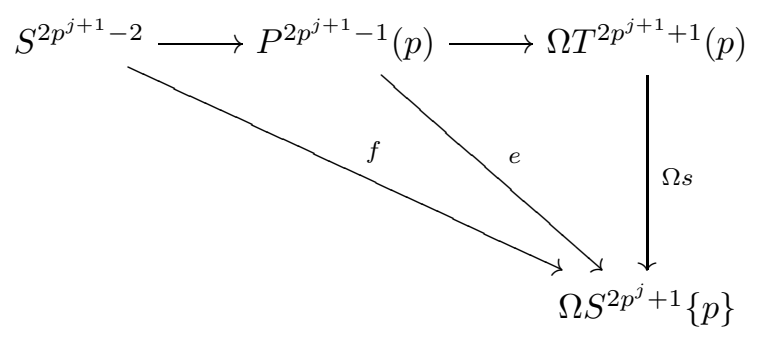

where the maps along the top row are skeletal inclusions, and hence $(\Omega s)_{*}$ is an isomorphism on $H_{2 p^{j+1}-2}()$ since $f_{*}$ is. Now since $H: \Omega S^{2 p^{j}+1}\{p\} \rightarrow B W_{p^{j}}$ induces an epimorphism in homology, the composite

$$
\Omega T^{2 p^{j+1}+1}(p) \stackrel{\Omega s}{\longrightarrow} \Omega S^{2 p^{j}+1}\{p\} \stackrel{H}{\longrightarrow} B W_{p^{j}}
$$

induces an isomorphism of the lowest nonvanishing reduced homology group

$$
H_{2 p^{j+1}-2}\left(\Omega T^{2 p^{j+1}+1}(p)\right) \cong H_{2 p^{j+1}-2}\left(B W_{p^{j}}\right) \cong \mathbb{Z} / p \mathbb{Z} .
$$

By [14, any map $\Omega T^{2 n p+1}(p) \rightarrow B W_{n}$ which is degree one on the bottom cell must be a homotopy equivalence, and thus $H \circ \Omega s$ is a homotopy equivalence. Composing a homotopy inverse of $H \circ \Omega s$ 
with $\Omega s$, we obtain a right homotopy inverse of $H$, which shows that the homotopy fibration

$$
T^{2 p^{j}+1}(p) \stackrel{E}{\longrightarrow} \Omega S^{2 p^{j}+1}\{p\} \stackrel{H}{\longrightarrow} B W_{p^{j}}
$$

splits. Moreover, letting $m$ denote the loop multiplication on $\Omega S^{2 p^{j}+1}\{p\}$, the composite

$$
T^{2 p^{j}+1}(p) \times \Omega T^{2 p^{j+1}+1}(p) \stackrel{E \times \Omega s}{\longrightarrow} \Omega S^{2 p^{j}+1}\{p\} \times \Omega S^{2 p^{j}+1}\{p\} \stackrel{m}{\longrightarrow} \Omega S^{2 p^{j}+1}\{p\}
$$

defines an equivalence of $H$-spaces since $E$ and $\Omega s$ are $H$-maps and $m$ is homotopic to the loops on the $H$-space multiplication on $S^{2 p^{j}+1}\{p\}$.

The homotopy equivalence $H \circ \Omega s: \Omega T^{2 p^{j+1}+1}(p) \rightarrow B W_{p^{j}}$ is not necessarily multiplicative, but the $H$-space decomposition of $\Omega S^{2 p^{j}+1}\{p\}$ constructed above can now be used exactly as in the proof of [26, Theorem 1.1] to produce an $H$-map $B W_{p^{j}} \rightarrow \Omega T^{2 p^{j+1}+1}(p)$ which is also a homotopy equivalence.

It remains to show that there is an equivalence of $H$-spaces $B W_{p^{j-1}} \simeq \Omega T^{2 p^{j}+1}(p)$. In his construction of a classifying space of $W_{n}$, Gray [11] introduced a $p$-local homotopy fibration

$$
B W_{n} \stackrel{j}{\longrightarrow} \Omega^{2} S^{2 n p+1} \stackrel{\phi}{\longrightarrow} S^{2 n p-1}
$$

where the map $j$ has order $p$ and hence lifts to a map $j^{\prime}: B W_{n} \rightarrow \Omega^{2} S^{2 n p+1}\{p\}$. By [25], $j^{\prime}$ can be chosen to be an $H$-map when $p \geq 3$. Since $j_{*}$ is an isomorphism in degree $2 n p-1$, it follows by commutativity with the Bockstein that $j_{*}^{\prime}$ is an isomorphism in degree $2 n p-2$. Let $\gamma$ denote the equivalence of $H$-spaces $T^{2 p^{j}+1}(p) \times \Omega T^{2 p^{j+1}+1}(p) \stackrel{\sim}{\longrightarrow} \Omega S^{2 p^{j}+1}\{p\}$ constructed above. As $\Omega \gamma$ is also an equivalence of $H$-spaces, it has a homotopy inverse $(\Omega \gamma)^{-1}$ which is also an $H$-map. Consider the composite

$$
B W_{p^{j-1}} \stackrel{j^{\prime}}{\longrightarrow} \Omega^{2} S^{2 p^{j}+1}\{p\} \stackrel{(\Omega \gamma)^{-1}}{\longrightarrow} \Omega T^{2 p^{j}+1}(p) \times \Omega^{2} T^{2 p^{j+1}+1}(p) \stackrel{\pi_{1}}{\longrightarrow} \Omega T^{2 p^{j}+1}(p)
$$

where $j^{\prime}$ is the lift of $j$ with $n=p^{j-1}$ and $\pi_{1}$ is the projection onto the first factor. Since all three maps in this composition induce isomorphisms on $H_{2 p^{j}-2}($ ), it again follows from the atomicity result in [14] that the composite defines a homotopy equivalence $B W_{p^{j-1}} \simeq \Omega T^{2 p^{j}+1}(p)$, which is an equivalence of $H$-spaces since each map above is an $H$-map.

\section{Applications}

In this section we derive Corollary 1.2 and Theorem 1.4 from Theorem 1.1 and discuss some other consequences in the $p=3$ case.

3.1. The homotopy decomposition of $\Omega S^{55}\{3\}$. Since, by [22, $\Omega S^{2 n+1}\{p\}$ is atomic for all $n$ such that $\pi_{2 n(p-1)-2}^{S}$ contains no element of $p$-primary Kervaire invariant one, $\Omega S^{2 n+1}\{p\}$ is indecomposable for $n \neq p^{j}$ and it follows from Theorem 1.1 that the decomposition problem for $\Omega S^{2 n+1}\{p\}$ is equivalent to the strong $p$-primary Kervaire invariant problem for odd primes $p$. The 3-primary Kervaire invariant problem is open, but the elements $b_{j-1} \in \operatorname{Ext}_{\mathcal{A}_{p}}^{2,2 p^{j}(p-1)}\left(\mathbb{F}_{p}, \mathbb{F}_{p}\right)$ in the $E_{2}$-term of the Adams spectral sequence which potentially detect elements of odd primary Kervaire invariant one are known to behave differently for $p=3$ than they do for primes $p \geq 5$.

While $b_{0}$ is a permanent cycle representing $\theta_{1} \in \pi_{2 p(p-1)-2}^{S}$ at all odd primes, Ravenel showed in [18] that for $j>1$ and $p \geq 5$ the elements $b_{j-1}$ support nontrivial differentials in the Adams 
spectral sequence and hence that none of the $\theta_{j}$ exist for $j>1$ and $p \geq 5$. For $p=3$, however, it is known (see [18, 19]) that, although $b_{1}$ supports a nontrivial differential, $b_{2}$ is a permanent cycle representing a 3 -primary Kervaire invariant class $\theta_{3} \in \pi_{106}^{S}$.

Proof of Corollary 1.2. According to [19], $\pi_{106}^{S} \cong \mathbb{Z} / 3 \mathbb{Z}$ after localizing at $p=3$, so $\theta_{3}$ has order 3 and the result follows from Theorem 1.1

Remark 3.1. We note that the nonexistence of $\theta_{2}$ at $p=3$ implies that $\Omega S^{2 p^{2}+1}\{p\}=\Omega S^{19}\{3\}$ is atomic and hence indecomposable by the result in 22 mentioned above.

Observe that since the mod $p$ Moore space $P^{2}(p)$ is the homotopy cofibre of the degree $p$ self map $p: S^{1} \rightarrow S^{1}$, by applying the functor $\operatorname{Map}_{*}\left(-, S^{2 n+1}\right)$ to the homotopy cofibration

$$
S^{1} \stackrel{p}{\longrightarrow} S^{1} \longrightarrow P^{2}(p)
$$

we obtain a homotopy fibration

$$
\operatorname{Map}_{*}\left(P^{2}(p), S^{2 n+1}\right) \longrightarrow \Omega S^{2 n+1} \stackrel{p}{\longrightarrow} \Omega S^{2 n+1}
$$

which identifies the mapping space $\operatorname{Map}_{*}\left(P^{2}(p), S^{2 n+1}\right)$ with the homotopy fibre $\Omega S^{2 n+1}\{p\}$ of the $p^{\text {th }}$ power map on the loop space $\Omega S^{2 n+1}$. The decomposition of $\Omega S^{55}\{3\}$ in Corollary 1.2 therefore induces the following splitting of homotopy groups with $\mathbb{Z} / 3 \mathbb{Z}$ coefficients analogous to Selick's [21] splitting of $\pi_{*}\left(S^{2 p+1} ; \mathbb{Z} / p \mathbb{Z}\right)$.

Corollary 3.2. For $k \geq 4$, there are isomorphisms

$$
\begin{aligned}
\pi_{k}\left(S^{55} ; \mathbb{Z} / 3 \mathbb{Z}\right) & \cong \pi_{k-2}\left(T^{55}(3)\right) \oplus \pi_{k-1}\left(T^{163}(3)\right) \\
& \cong \pi_{k-4}\left(W_{9}\right) \oplus \pi_{k-3}\left(W_{27}\right) .
\end{aligned}
$$

3.2. Homotopy associativity and exponents for mod 3 Anick spaces. The following two useful properties of $T^{2 n+1}(p)$ were conjectured by Anick and Gray [2, 3]:

(i) $T^{2 n+1}(p)$ is a homotopy commutative and homotopy associative $H$-space;

(ii) $T^{2 n+1}(p)$ has homotopy exponent $p$.

Both properties have been established for all $p \geq 5$ and $n \geq 1$, but only partial results have been obtained in the $p=3$ case. For example, it was found in [24 that $T^{7}(3)$ is both homotopy commutative and homotopy associative but that homotopy associativity fails for $T^{11}(3)$. More generally, Gray showed in [13] that if $T^{2 n+1}(3)$ is homotopy associative, then $n=3^{j}$ for some $j \geq 0$ and moreover that property (i) implies property (ii).

Concerning property (ii), in general $T^{2 n+1}(3)$ is only known to have homotopy exponent bounded above by 9 . (This can be seen using fibration (2) and the fact that $B W_{n}$ has 3-primary exponent 3 , for example.) Since $T^{2 n+1}(p)$ is an $H$-space for all $p \geq 3$, one could also ask for the stronger property that $T^{2 n+1}(p)$ has $H$-space exponent $p$, i.e., that its $p^{\text {th }}$ power map is null homotopic. We note that, when they occur, decompositions of $\Omega S^{2 n+1}\{3\}$ give some evidence for (ii). 
Corollary 3.3. The following hold:

(a) $T^{7}(3)$ and $T^{55}(3)$ are homotopy commutative and homotopy associative $H$-spaces;

(b) $T^{7}(3), T^{55}(3), \Omega T^{19}(3)$ and $\Omega T^{163}(3)$ each have $H$-space exponent 3 .

Proof. Since the homotopy equivalences $\Omega S^{7}\{3\} \simeq T^{7}(3) \times \Omega T^{19}(3)$ and $\Omega S^{55}\{3\} \simeq T^{55}(3) \times$ $\Omega T^{163}(3)$ which follow from Theorem 1.1 are equivalences of $H$-spaces, part (b) follows immediately from the fact that $\Omega S^{2 n+1}\{3\}$ has $H$-space exponent 3 [17, and part (a) follows from the fact that $\Omega S^{2 n+1}\{3\}$ is homotopy associative and homotopy commutative as it is the loop space of an $H$-space.

Proof of Theorem 1.4. Let $n>1$ and suppose $T^{2 n+1}(3)$ is homotopy associative. Then the proof of [13, Theorem A.2] shows that there exists a three-cell complex

$$
X=S^{2 n+1} \cup_{3} e^{2 n+2} \cup e^{6 n+1}
$$

with nontrivial mod 3 Steenrod operation $\mathcal{P}^{n}: H^{2 n+1}(X) \rightarrow H^{6 n+1}(X)$. The attaching map of the middle cell of a Spanier-Whitehead dual of $X$ then defines an element of Kervaire invariant one in $\pi_{4 n-2}^{S}$ which has order 3 since it extends over a mod 3 Moore space. Alternatively, by [24, Proposition 7.1], the homotopy associativity of $T^{2 n+1}(3)$ implies that a certain composite

$$
S^{6 n-3} \stackrel{[\iota,[\iota, \iota]]}{\longrightarrow} \Omega S^{2 n} \stackrel{r}{\longrightarrow} S^{2 n-1}
$$

is divisible by 3 , where $[\iota,[\iota, \iota]]$ denotes the triple Samelson product of the generator of $\pi_{2 n-1}\left(\Omega S^{2 n}\right)$ and $r$ is a left homotopy inverse of the suspension $E: S^{2 n-1} \rightarrow \Omega S^{2 n}$. It is easy to check that the composite above coincides with the image of the generator of the lowest nonvanishing 3-local homotopy group $\pi_{6 n-3}\left(W_{n}\right) \cong \mathbb{Z} / 3 \mathbb{Z}$ under the homotopy fibre map $W_{n} \rightarrow S^{2 n-1}$, and the divisibility of this element is a well-known equivalent formulation of the strong Kervaire invariant problem.

Conversely, if there exists a 3-primary Kervaire invariant element of order 3 in $\pi_{4 n-2}^{S}$, then $n=3^{j}$ for some $j \geq 1$ and it follows from Theorem 1.1 that $T^{2 n+1}(3)$ is a homotopy associative $H$-space as it is an $H$-space retract of a loop space.

\section{A stable SPLitting of $\Omega S^{2 n+1}\{p\}$}

It is well known that $S^{2 n+1}\{p\}$ splits as a wedge of $\bmod p$ Moore spaces after suspending once. In this section we determine the stable homotopy type of the loop space $\Omega S^{2 n+1}\{p\}$ by observing that although the homotopy fibration

$$
T^{2 n+1}(p) \stackrel{E}{\longrightarrow} \Omega S^{2 n+1}\{p\} \stackrel{H}{\longrightarrow} B W_{n}
$$

only splits in Kervaire invariant one dimensions, it splits for all $n$ after suspending twice. As in the previous sections, $p$ denotes an odd prime and all spaces and maps are assumed to be localized at $p$.

Proposition 4.1. For all $n \geq 1$, there is a homotopy equivalence

$$
\Sigma^{2} \Omega S^{2 n+1}\{p\} \simeq \Sigma^{2}\left(T^{2 n+1}(p) \times B W_{n}\right) .
$$


Proof. In 11], Gray showed that the classifying space $B W_{n}$ of the fibre of the double suspension fits in a homotopy fibration

$$
S^{2 n-1} \stackrel{E^{2}}{\longrightarrow} \Omega^{2} S^{2 n+1} \stackrel{\nu}{\longrightarrow} B W_{n}
$$

and that there is a homotopy equivalence $\Sigma^{2} \Omega^{2} S^{2 n+1} \simeq \Sigma^{2}\left(S^{2 n-1} \times B W_{n}\right)$. Let $s: \Sigma^{2} B W_{n} \rightarrow$ $\Sigma^{2} \Omega^{2} S^{2 n+1}$ be a right homotopy inverse of $\Sigma^{2} \nu$. In the construction of Anick's fibration in [15], $T^{2 n+1}$ is defined as the homotopy fibre of the map $H$, where $H$ is constructed as an extension

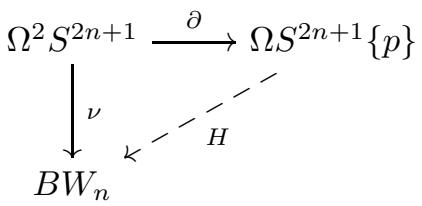

of $\nu$ through the connecting map of the homotopy fibration $\Omega S^{2 n+1}\{p\} \longrightarrow \Omega S^{2 n+1} \stackrel{p}{\longrightarrow} \Omega S^{2 n+1}$. Therefore, by composing $s$ with $\Sigma^{2} \partial$, we obtain a right homotopy inverse $s^{\prime}: \Sigma^{2} B W_{n} \rightarrow \Sigma^{2} \Omega S^{2 n+1}\{p\}$ of $\Sigma^{2} H$. Next, consider the composite map $f$ defined by

$$
f: T^{2 n+1}(p) \wedge \Sigma^{2} B W_{n} \stackrel{E \wedge s^{\prime}}{\longrightarrow} \Omega S^{2 n+1}\{p\} \wedge \Sigma^{2} \Omega S^{2 n+1}\{p\} \longrightarrow \Sigma^{2} \Omega S^{2 n+1}\{p\}
$$

where the second map is obtained by suspending the Hopf construction $\Sigma \Omega S^{2 n+1}\{p\} \wedge \Omega S^{2 n+1}\{p\} \rightarrow$ $\Sigma \Omega S^{2 n+1}\{p\}$ on $\Omega S^{2 n+1}\{p\}$. Finally, since $\Sigma^{2} E, s^{\prime}$ and $f$ each induce monomorphisms in $\bmod p$ homology, it follows that the map

$$
\Sigma^{2}\left(T^{2 n+1}(p) \times B W_{n}\right) \simeq \Sigma^{2} T^{2 n+1}(p) \vee \Sigma^{2} B W_{n} \vee\left(\Sigma^{2} T^{2 n+1}(p) \wedge B W_{n}\right) \longrightarrow \Sigma^{2} S^{2 n+1}\{p\}
$$

defined by their wedge sum is a homology isomorphism and hence a homotopy equivalence.

It follows from Proposition 4.1 that $\Omega S^{2 n+1}\{p\}$ has the stable homotopy type of a wedge of Moore spaces, Snaith summands $D_{2, k}\left(S^{2 n-1}\right)$ of the stable splitting of $\Omega^{2} S^{2 n+1}$, and their smash products.

A similar argument can be used to give a stable splitting of the homotopy fibre $E^{2 n+1}$ of the natural inclusion $i: P^{2 n+1}(p) \rightarrow S^{2 n+1}\{p\}$ where $B W_{n}$ is a stable retract. More precisely, it follows from [15] that the extension $H$ of $\nu$ appearing in the proof of Proposition 4.1 can be chosen to factor through a map $\delta: \Omega S^{2 n+1}\{p\} \rightarrow E^{2 n+1}$, and thus $B W_{n}$ also retracts off $E^{2 n+1}$ after suspending twice. The space $E^{2 n+1}$ along with a homotopy pullback diagram

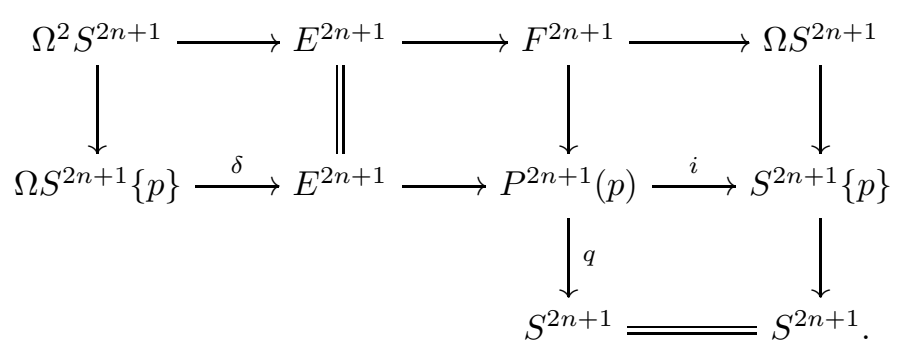

determined by the factorization of the pinch map $q: P^{2 n+1}(p) \rightarrow S^{2 n+1}$ in the bottom right square was thoroughly analyzed in Cohen, Moore and Neisendorfer's study of the homotopy theory of Moore spaces [6, 7], where decompositions of $\Omega E^{2 n+1}, \Omega F^{2 n+1}$ and $\Omega P^{2 n+1}(p)$ were used to determine the homotopy exponents of spheres and Moore spaces. The double suspension fibration $W_{n} \longrightarrow$ $S^{2 n-1} \stackrel{E^{2}}{\longrightarrow} \Omega^{2} S^{2 n+1}$ was shown to retract off the homotopy fibration along the top row of the loops 
on (3) with $W_{n}$ and $S^{2 n-1}$ appearing as factors containing the bottom cells in product decompositions of $\Omega E^{2 n+1}$ and $\Omega F^{2 n+1}$, respectively.

Consider the morphism of homotopy fibrations

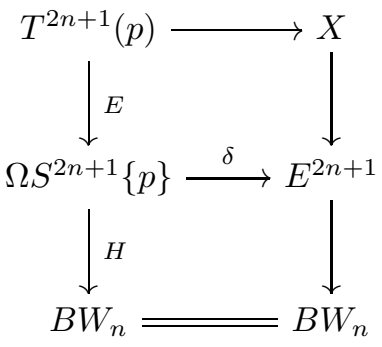

determined by the factorization of $H$ through $\delta$. As with the splitting in Proposition 4.1, the retraction of $\Sigma^{2} B W_{n}$ off $\Sigma^{2} E^{2 n+1}$ can be desuspended in Kervaire invariant one dimensions. One difference, however, is that since $W_{n}$ is always a retract of $\Omega E^{2 n+1}$ by Cohen, Moore and Neisendorfer's decomposition, the image of the homology class $b_{2 n p-2} \in H_{2 n p-2}\left(\Omega S^{2 n+1}\{p\}\right)$ under $\delta_{*}$ is spherical for all $n$ (as opposed to just those $n=p^{j}$ for which $\pi_{2 n(p-1)-2}^{S}$ contains an element of strong Kervaire invariant one), and thus the nonexistence of Kervaire invariant elements does not obstruct the possibility of an unstable decomposition of $E^{2 n+1}$ as it does for $\Omega S^{2 n+1}\{p\}$. It would therefore be interesting to know if a homotopy class $S^{2 n p-2} \rightarrow E^{2 n+1}$ with Hurewicz image $\delta_{*}\left(b_{2 n p-2}\right)$ could be extended to a map $\Omega T^{2 n p+1}(p) \rightarrow E^{2 n+1}$ as in the proof of Theorem 1.1 to prove the conjectured homotopy equivalence $B W_{n} \simeq \Omega T^{2 n p+1}(p)$ for all $n$ and split the homotopy fibration in the second column of the diagram above. We show below that $B W_{n}$ is in fact a retract of $E^{2 n+1}$ for all $n$, delooping the result of Cohen, Moore and Neisendorfer.

Proposition 4.2. For all $n \geq 1, B W_{n}$ is a retract of $E^{2 n+1}$.

Proof. By the construction of $B W_{n}$ in [11, there is a homotopy fibration sequence

$$
\Omega^{2} S^{2 n+1} \longrightarrow B W_{n} \times S^{4 n-1} \longrightarrow S^{2 n} \stackrel{E}{\longrightarrow} \Omega S^{2 n+1}
$$

where the connecting map factors as $\Omega^{2} S^{2 n+1} \stackrel{\nu}{\longrightarrow} B W_{n} \stackrel{i_{1}}{\longrightarrow} B W_{n} \times S^{4 n-1}$. Since the composite $S^{2 n} \stackrel{E}{\longrightarrow} \Omega S^{2 n+1} \longrightarrow S^{2 n+1}\{p\}$ is just the inclusion of the bottom cell of $S^{2 n+1}\{p\}$, there is a homotopy commutative diagram

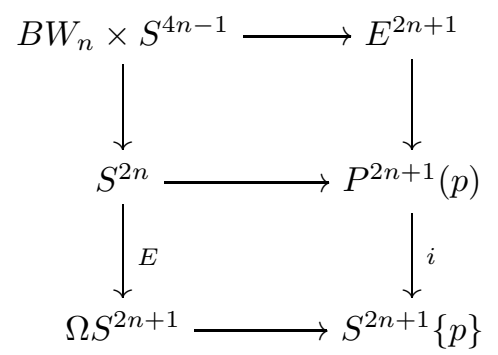

where the induced map of fibres determines a map $g: B W_{n} \rightarrow E^{2 n+1}$. Observe that the connecting map $\Omega^{2} S^{2 n+1} \rightarrow B W_{n} \times S^{4 n-1}$ of the first column induces an isomorphism on $H_{2 n p-2}($ ) since $\nu$ does, and the connecting map $\delta: \Omega S^{2 n+1}\{p\} \rightarrow E^{2 n+1}$ of the second column induces an isomorphism on $H_{2 n p-2}$ ( ) by the commutativity of (4). Therefore, since the map $\Omega^{2} S^{2 n+1} \rightarrow \Omega S^{2 n+1}\{p\}$ given by 
the loops on the bottom horizontal map induces a monomorphism in homology by a Serre spectral sequence argument, we conclude that $g: B W_{n} \rightarrow E^{2 n+1}$ induces an isomorphism on $H_{2 n p-2}()$ so that the composition $B W_{n} \stackrel{g}{\rightarrow} E^{2 n+1} \rightarrow B W_{n}$ with the extension in (4) is degree one on the bottom cell and thus a homotopy equivalence.

\section{REFERENCES}

[1] S. Amelotte, A homotopy decomposition of the fibre of the squaring map on $\Omega^{3} S^{17}$, Homology, Homotopy and Applications 20 (2018), 141-154.

[2] D. Anick, Differential algebras in topology, Research Notes in Mathematics, AK Peters, 1993.

[3] D. Anick and B. Gray, Small H-spaces related to Moore spaces, Topology 34 (1995), 859-881.

[4] H. E. A. Campbell, F. R. Cohen, F. P. Peterson and P. S. Selick, The space of maps of Moore spaces into spheres, Proc. of John Moore Conf. on Alg. Top. and Alg. K-Theory, 72-100, Ann. Math. Studies vol. 113, Princeton Univ. Press, Princeton, 1987.

[5] F. R. Cohen, Two-primary analogues of Selick's theorem and the Kahn-Priddy theorem for the 3-sphere, Topology 23 (1984), 401-421.

[6] F. R. Cohen, J. C. Moore and J. A. Neisendorfer, Torsion in homotopy groups, Ann. Math. 109 (1979), 121-168.

[7] F. R. Cohen, J. C. Moore and J. A. Neisendorfer, The double suspension and exponents of the homotopy groups of spheres, Ann. Math. 110 (1979), 549-565.

[8] F. R. Cohen, J. C. Moore and J. A. Neisendorfer, Decompositions of loop spaces and applications to exponents, Alg. Top., Proc. Sympos., Univ. Aarhus, Aarhus, 1978, 1-12, Lecture Notes in Math., 763, Springer, Berlin, 1979.

[9] F. R. Cohen and P. S. Selick, Splittings of two function spaces, Quart. J. Math. Oxford 41 (1990), 145-153.

[10] B. Gray, On the sphere of origin of infinite families in the homotopy groups of spheres, Topology 8 (1969), $219-232$.

[11] B. Gray, On the iterated suspension, Topology 27 (1988), 301-310.

[12] B. Gray, EHP spectra and periodicity. I. Geometric constructions, Trans. Amer. Math. Soc. 340 (1993), 595-616.

[13] B. Gray, Abelian properties of Anick spaces, Mem. Amer. Math. Soc. 246 (2017).

[14] B. Gray and S. Theriault, On the double suspension and the mod-p Moore space, Contemp. Math. 399 (2006), 101-121.

[15] B. Gray and S. Theriault, An elementary construction of Anick's fibration, Geom. Topol. 14 (2010), $243-275$.

[16] J. A. Neisendorfer, 3-primary exponents, Math. Proc. Cambridge Philos. Soc. 90 (1981), 63-83.

[17] J. A. Neisendorfer, Properties of certain H-spaces, Quart. J. Math. Oxford 34 (1983), 201-209.

[18] D. C. Ravenel, The non-existence of odd primary Arf invariant elements in stable homotopy, Math. Proc. Cambridge Philos. Soc. 83 (1978), 429-443.

[19] D. C. Ravenel, Complex Cobordism and Stable Homotopy Groups of Spheres, second ed., AMS Chelsea Publishing, vol. 347, Amer. Math. Soc., Providence, RI, 2004.

[20] P. S. Selick, Odd primary torsion in $\pi_{k}\left(S^{3}\right)$, Topology 17 (1978), 407-412.

[21] P. S. Selick, A decomposition of $\pi_{*}\left(S^{2 p+1} ; \mathbb{Z} / p \mathbb{Z}\right)$, Topology 20 (1981), 175-177.

[22] P. S. Selick, A reformulation of the Arf invariant one mod p problem and applications to atomic spaces, Pac. J. Math. 108 (1983), 431-450.

[23] P. S. Selick, Space exponents for loop spaces of spheres, Fields Inst. Commun. 19, Amer. Math. Soc., 1998, $279-283$.

[24] S. Theriault, Properties of Anick's spaces, Trans. Amer. Math. Soc. 353 (2001), 1009-1037.

[25] S. Theriault, The 3-primary classifying space of the fiber of the double suspension, Proc. Amer. Math. Soc. 136 (2008), 1489-1499.

[26] S. Theriault, A case when the fiber of the double suspension is the double loops on Anick's space, Can. Math. Bull. 53 (2010), 730-736.

E-mail address: steven.amelotte@rochester.edu 